\title{
A Testbed Design of Spectrum Management in Cognitive Radio Network using NI USRP and LabVIEW
}

\author{
Vinod H. Patil, Shruti Oza, Vishal Sharma, Asrithasiripurapu, Tejaswini Patil
}

\begin{abstract}
Cognitive radio automatically detects the available channel in the wireless communication and has an adaptive radio technology network. It also changes the transmission parameters to run concurrently for more smooth communication. CR network allows the user to utilize the band in an opportunistic manner because it has different characteristics like capabilities and re-configurability. In this article, it shown the methodology, on how the primary user and the secondary user should communicate to provide error free communication. And also the framework on how to overcome the unique challenges occurred in the spectrum management like interference avoidance, QoS awareness and seamless communication. We also discussed about spectrum mobility, spectrum sharing, spectrum decision, and also spectrum sensing which are the characteristics of spectrum management.
\end{abstract}

Keywords: Cognitive Radio (CR), Quality of Service (QoS), Primary User (PU), Secondary User (SU).

\section{INTRODUCTION}

Spectrum is known as a band of frequencies. There are two extreme points between which classification of discrete frequencies is defined in terms of its position on a scale.

Due to increasing in demand for wireless broadband, such as $3 \mathrm{G}$ and $4 \mathrm{G}$ mobile services, spectrum management was introduced. Spectrum is assigned through administrative licensing since 1930. Signal interference was considered as a major problem for spectrum users. Therefore, licensing was established to reject signal interference.

The main aim of this project is to use the unused part of spectrum of licensed user or primary user by unlicensed user or secondary user. Licensed user has authority to share its spectrum with unlicensed. The only condition is that licensed user is not using a band of frequencies so that unlicensed user can request them and can use those unused band of frequencies.

Revised Version Manuscript Received on August 19, 2019.

Mr.Vinod H. Patil, Department of Electronics and Telecommunication Engineering, BharatiVidyapeeth (Deemed to be University) College of Engineering, Pune, Maharashtra, India.(email: : vhpatil@bvucoep.edu.in)

Dr.ShrutiOza, Department of Electronics and Telecommunication Engineering, BharatiVidyapeeth (Deemed to be University) College of Engineering, Pune, Maharashtra, India(email: : skoza@bvucoep.edu.in)

Vishal Sharma, Department of Electronics and Telecommunication Engineering, BharatiVidyapeeth (Deemed to be University) College of Engineering, Pune, Maharashtra, India

Asrithasiripurapu, Department of Electronics and Telecommunication Engineering, BharatiVidyapeeth (Deemed to be University) College of Engineering, Pune, Maharashtra, India

TejaswiniPatil, Department of Electronics and Telecommunication Engineering, BharatiVidyapeeth (Deemed to be University) College of Engineering, Pune, Maharashtra, India
Here we managed spectrum by modifying it and utilizing it. It is the process to regulate the use of radio frequencies. It promotes efficient use and gain of radio frequencies. The range of radio frequency is from $3 \mathrm{KHZ}$ to $300 \mathrm{GHZ}$. It is used for wireless communication. For this spectrum management, we used cognitive radio. Cognitive radio is a smart radio technology which has the capability to take smart decisions itself. It is used to detect the unused part of bandwidth of the primary user's spectrum.

Cognitive Radio provides the capability to share the wireless channel with licensed user in an opportunistic manner. Because of high variations in the spectrum and of QoS applications, the CR network has unusual problems. Thus, to label these problems, each secondary user in the CR network must have: -

1) To find the availability of the portion in the spectrum.

2) To select the channel, this is available after checking the availability.

3) To access the channels for different users.

4) The channel should be available when the primary user needs [2].

These challenges of spectrum management can be managed by the following four important functions known as: Sensing of spectrum, decision of spectrum, sharing of spectrum, and mobility of spectrum.

We will also learn about some functions and other research motive in this article. We will also discuss about our further research development of Cognitive Radio networks which requires no modifications in the present network. The brief description of Cognitive Radio network technology is described, and also the architecture of Cognitive Radio network is provided.

To overcome the spectrum unused problem, various measures or steps are taken by the licensed operator like, the primary user or licensed user allows the multiple secondary users or unlicensed users to divert their traffic through their spectrum by taking the frequency of that spectrum on rent for a fixed duration. 


\section{METHODOLOGY}

a) Cognitive Radio Network

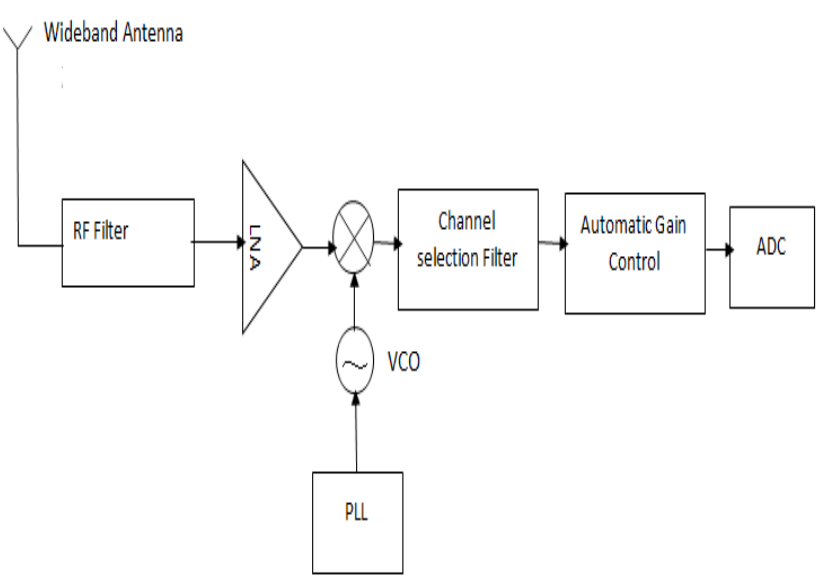

Fig.1. Block diagram of Cognitive Radio

Figure 1 shows the basic cognitive radio uses trans-receiver which consists of various functions such as

- RF filter:

RF filter removes the undesired frequency and selects the desired frequency.

\section{- Low noise amplifier (LNA):}

Low Noise Amplifier amplifies the input frequency so that we can determine the precise value of the noise figure of the input signal.

- Mixer:

Mixers are used to convert the high frequency signals into the low frequency signals i.e, Intermediate frequency signals, to make it eligible for the further process. This also allows making the low power filters, which helps to study about the low power signals and noises.

- Voltage controlled oscillator (VCO):

It is used to make the mixed frequency signals from the multiple frequencies.

- Phase locked loop (PLL):

Phase Locked Loop checks whether the frequency is constant as same as the time.

- Channel selection filter:

This filter selects the wanted frequency band and then rejects the next frequency signal

- Automatic gain control(AGC):

Over a great distance of input signal, the controller keeps the output power constant.

- $A / D$ converter $(A D C)$ :

In analog to digital converter, the steps like quantization, encoding etc., takes place, which can leads to understand by the baseband signals generated at the baseband processing units.

\section{b) Architecture:}

The components of the Cognitive Radio network is divided in two groups
Primary network user (licensed user) and the CR network user (unlicensed user)

Then primary network or the licensed network, have the authority to operate in a given frequency band as they have buoyed the spectrum. If primary networks have any infrastructure then the primary user activities are controlled through primary base stations. The operations of primary users should not be affected by any unlicensed user because of their priorities in spectrum access.

The CR network cannot operate in given spectrum band, as it has no authority (no license). Thus, CR user requires more functions to share the licensed spectrum. Finally, CR networks include some negotiator who negotiates the spectrum for different CR users.

The main functions of licensed user and the unlicensed user are defined as:

\section{- Primary user:}

It should not get effected by any unlicensed user as only the licensed user have the authority to operate and access the spectrum band

\section{- Primary Base-Station:}

The main principle of base station is not having ability to share the spectrum with secondary users. To allot frequency to the secondary or CR users the primary user must have the legacy and CR protocols. Thus, the primary has the licensed spectrum which makes it a fixed infrastructure network.

\section{- Cognitive radio user:}

his user does not have the licensed spectrum which is why it has only few limits to use the spectrum. CR user assumed for having the abilities not only to communicate with both base station and other secondary users. These capabilities also include sensing, sharing, and decision.

\section{- Cognitive radio Base-Station:}

Even though there is no access over the spectrum, this base station provides the single hop connection.

CR user has different access over which the network can implement as it has to communicate with each other in either multi hop or access from base station manner.

\section{- Cognitive radio network access:}

The medium access plan is to be independent of that of primary network as the interactions took place inside the CR network. Thus, CR user can ingress in authorized network and also in unauthorized network. 


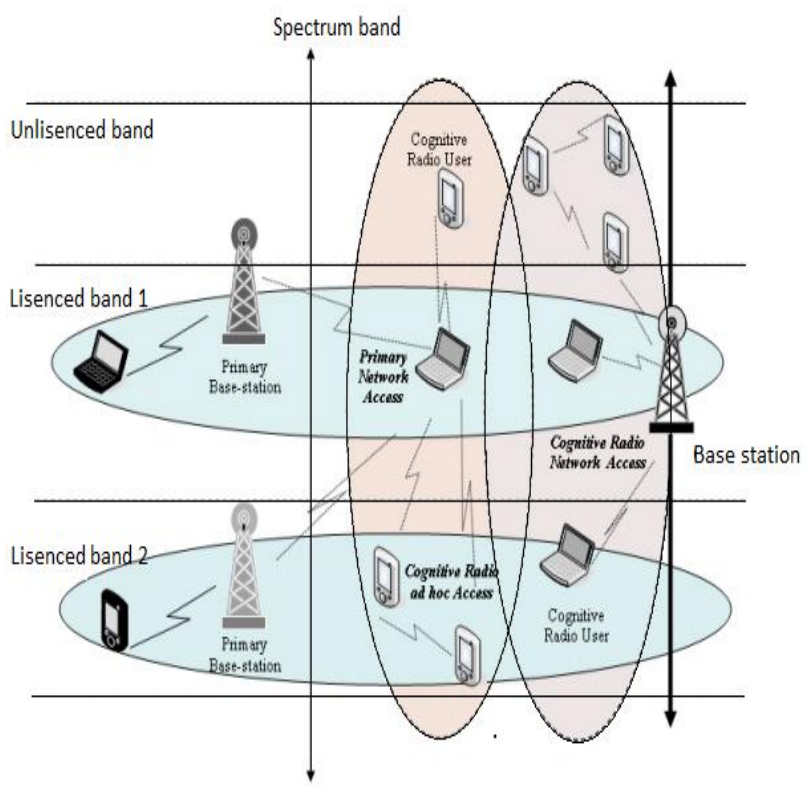

Fig.2. Architecture of Cognitive Radio

- Cognitive radio Ad Hoc access:

Ad Hoc access is same as the network access. But in network access the ingress is done through medium plans whereas in this the communication is done by Ad Hoc connection and can have their own medium technology access.

\section{- Primary network access:}

If primary network is allowed, the access to the primary base station is done through licensed spectrum by CR users. CR user supports the medium access technology of primary network unlike other users. Furthermore, primary base station should support CR capabilities.

\section{c) Framework of Spectrum Management:}

As discussed previously the CR network impose unique challenge because of its fact of living with different QoS resources. Thus, the spectrum management functions are used for Cognitive Radio networks for following challenges:

\section{- Interference avoidance:}

there should be no interference or any disturbance between primary user and the CR user.

\section{- QoS awareness:}

to decide the spectrum, the CR user supports the Quality of service awareness.

\section{- Seamless communication:}

CR network should provide smooth communication for the appearance of primary users.

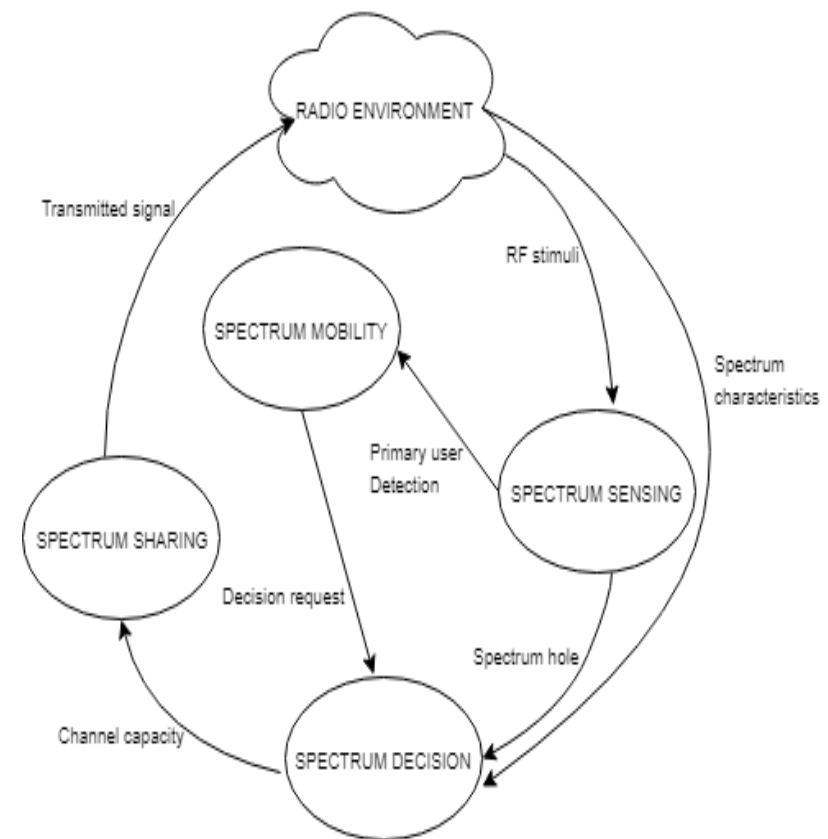

Fig.3. Framework of Spectrum Management

The spectrum management process consists of four major steps:

1. Spectrum sensing:

A CR user has sensed the available spectrum by monitoring, gathering data and by identifying holes.

2. Spectrum decision:

Once the band is sensed then CR user selects the best band according to their Quality of service [5].

3. Spectrum sharing:

As there are many CR users, the spectrum band should be shared equally to prevent colliding.

4. Spectrum mobility:

If any portion of the spectrum is required by the primary user which in use by the CR user, then the communication has been persist in other vacant portion.

\section{EXPERIMENTAL DETAILS}

\section{a) Hardware (NI USRP 2901)}

Ettus Research and its parent company designed a radios designed and sold, and the name of that software is Universal Software Radio Peripheral (USRP).It is generally used by research labs, universities, and hobbyists because it is intended be a competitively cheap hardware platform.

Universal Software Radio Peripheral (USRP), are generally connected with a high speed link to get in the connection with the host computer's software which controls all the functionalities of the USRP. There are various types of USRP's, with various functionalities. Some have a inbuilt hardware which have a inbuilt predefined processor which works in such a way that it works in its standalone state.

These devices are manufactured for approachable. The best part about this software is that it is open source codes and the software which is UHD is also open free to download. GNU Radio are basically used for the complex radio designs and system. 


\section{Design}

The USRP provides variety in models which works on the same architecture. Ait provides us various approaches like FPGA's, host processor, DAC's, power regulations, clock generations, ADCs and host interfacing. There is a front end board called daughter board which is used in various operations by a user's like filtering, up/down conversion and other kinds of front end variations.

There is a FPGA which provides the user to a different point of views for different kinds of DSP operations which basically attenuates the real signals transferred through analogy medium to low bit rates, with desired basebands in the digital premises. In various approaches, the UHD software present on the host computer to transfer the high bit rate DSP techniques and methods and also FPGA is freely available to all can be modified in accordance to our need like high speed.

\section{Software:}

The USPR hardware driver (UHD) is the software used to controls the functionality of the USRP. It also works well in various environments like Linux, Windows MacOS. UHD uses various frameworks like GNU Radio, LabVIEW, and SIMULINK. It also provides a support to $\mathrm{C}$ and $\mathrm{C}++$ functionalities, because it also supports their libraries too. e.g the UHD is also provides support to all kinds of USRPs by adjusting the variations of the input parameters in the USRPS.

LabVIEW supports all kinds of USRP interface like USRP1, USRP2 etc. and this also goes with the Simulink. They contain libraries which supports by both the software.

\section{NI USRP 2901 Specifications}

\section{Trans receiver}

Frequency range $70 \mathrm{MHz}$ to $6 \mathrm{GHz}$

Maximum output power $20 \mathrm{dBm}$

Bandwidth $56 \mathrm{MHz}$

One channel $61.44 \mathrm{MS} / \mathrm{s}$

Two channel $30.72 \mathrm{MS} / \mathrm{s}$

Digital-to-Analog converter (ADC) 512bits

Power:-

Total power, typical operation

Typical $3 \mathrm{~W}$ to $3.5 \mathrm{~W}$

Maximum $4.5 \mathrm{~W}$

Power requirement accepts a $6 \mathrm{~V}, 3 \mathrm{~A}$ external DC connector

\section{b) Software (LabVIEW)}

Laboratory Virtual Instrument Engineering Workbench (LabVIEW) is a platform which helps the users to design their projects with the help of the visual representation and also uses the graphical representation.

LabVIEW is basically comes into the picture when we want to acquisition of the data, to control the instruments, and also industrial automation in various operating systems like Windows, MacOS, Linux etc.

\section{Dataflow programming:}

The dataflow programming is the primary basic approach of the LabVIEW. In this, program is defined by the graphical block diagram representation. It consist wires which used to connects different nodes for the transfer of data, through different blocks.it also allows us to implements the front view like input output and also implement the further improvements.

\section{Graphical programming:}

LabVIEWworks as it allows the users to create different VI's which nothing is but virtual environment, which has a certain set of blocks combining and comes out as a working project. Every VI contains three different panels, a block panel, a front panel and a connection panel. The block panel contains the block diagram of the project and contains the functionalities, the libraries and codes which are responsible for the calculations of the logic and algorithms. This software also provides user to control the output by providing output window where the user can put the values with respect to the desired output.

This type to graphical approach also allows the non-programmer users to use this software easily. The advantage of this software is that it allows the user to make stand-alone projects. It also allows tracking the data flow of the project. Also, user can track the real time response with the high accuracy of the data and with high data speed.

\section{EXPERIMENTAL WORKFLOW}

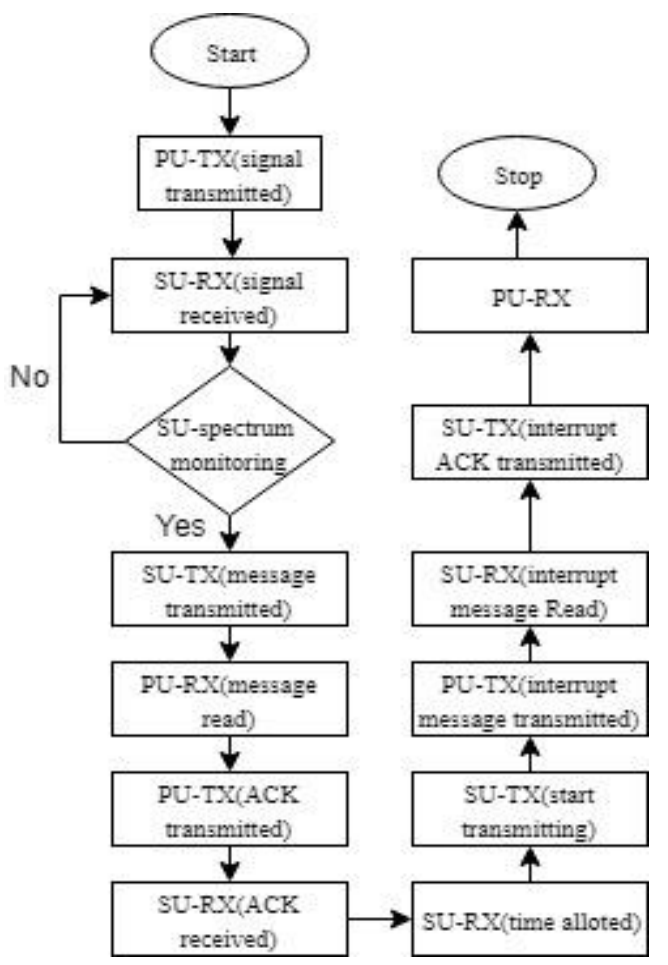

Fig.4. flowchart of the project

Time Allocation Flowchart

- As shown in fig.5., the acknowledgement request is received by the primary user, and then primary user system initially checks that the received string is empty or not, if it is empty then it do nothing, otherwise it starts checking the index of the string.

- Then, after finding out the first index of the string, 
system starts comparing that index with the $U$ which is the starting indentation of the string which help the system and the user to understand the start of the address, and then if they both proves unequal, then it states that it contains invalid information, otherwise, it proceeds to find the next index of that string.

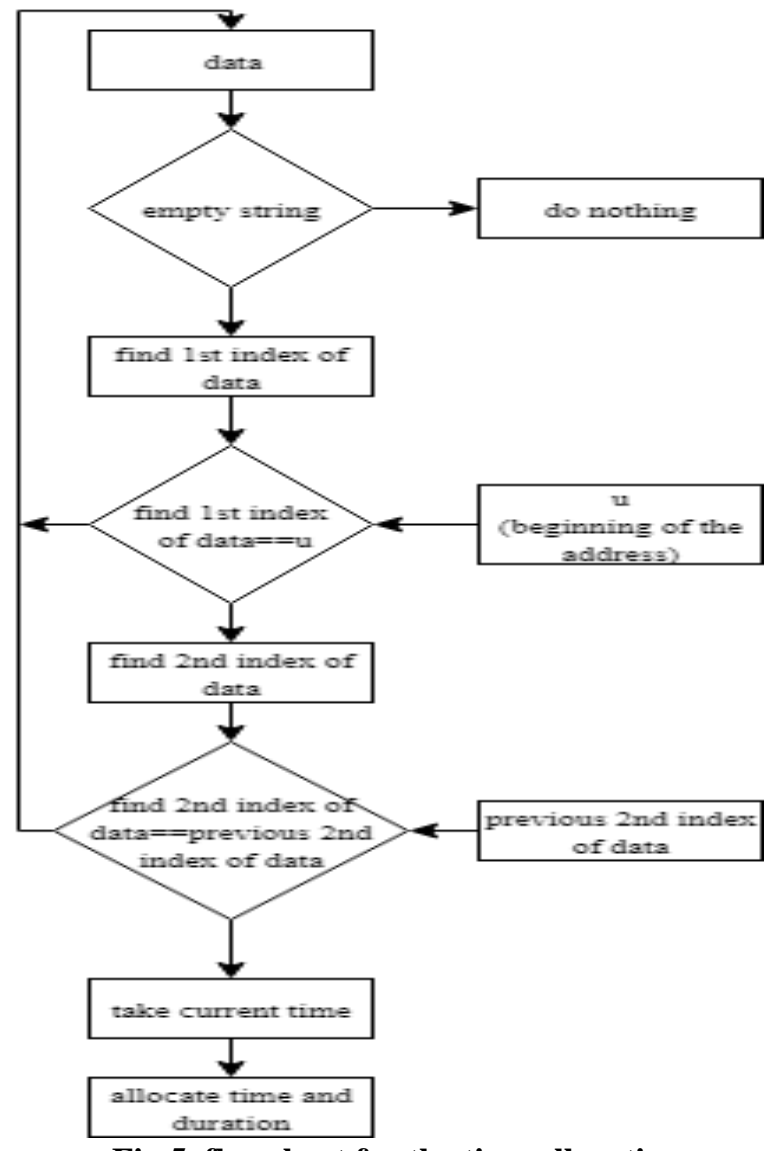

Fig.5. flowchart for the time allocation

- Now, the system compares the second index of the input string with the second index of the previous data's second index. If they prove equal then that means the input data is repeated and it will get terminated, otherwise it proves unique.

- After extracting the address of the secondary user, the primary system allocates the duration and time for the further session.

\section{EXPERIMENTAL SETUP}
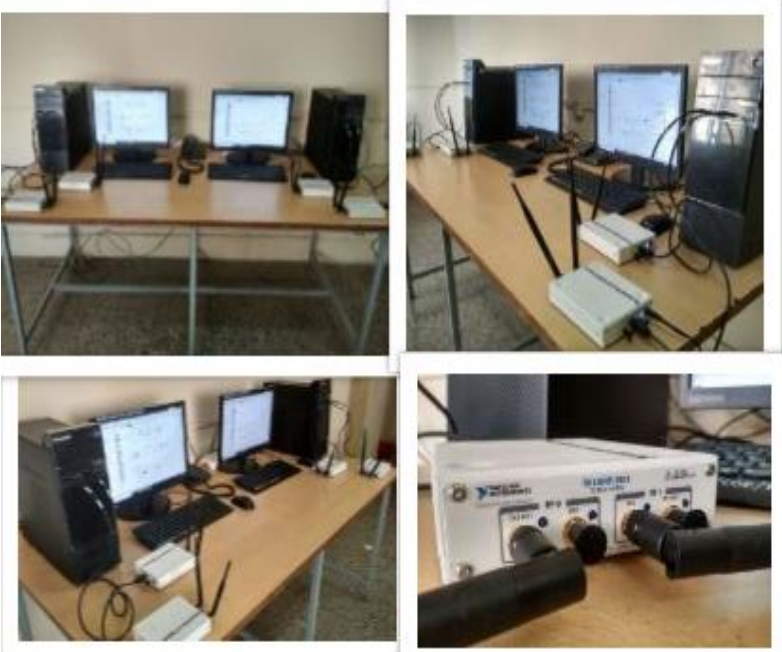

In our research, we have taken two users, the first one is the Primary User also we can name this the licensed user, which is considered as the high priority user and the other one is the Secondary User also we can say that the unlicensed user, which is considered as the low priority user as compared to the licensed one. For the communication between these two users we set up our system in which we used the frequency of $915 \mathrm{MHz}$ and also set the desired values of the gain, IQ rate, samples etc in accordance to our desired output. The system we used for our research, is of intel i5 generation, 8GB RAM. The USRPs we used require the power supply of $5 \mathrm{~W}$ and also to increase the range of the antennas we connect main power supply. For data transfer between the system and the USRP, we connected the USRP with the 3.0 port of the system. We used the vertical 900 antenna for our research which connected in the USRPs both ports (first one is Tx/Rx port, second one is only for $\mathrm{Rx}$ ). The vertical antenna we used of peak gain $+2 \mathrm{DB}$, operating temperature of $-20{ }^{\circ} \mathrm{C}$ to $+65^{\circ} \mathrm{Cand}$ a frequency range of $\sim 902-928 \mathrm{MHz}$ centred at $915 \mathrm{MHz}$.

\section{EXPERIMENTAL RESULTS}

\section{a) Primary user transmit the signal}

We use USRP 2901 as a both primary and receiver. The setup is designed in such a way that the when the primary is transmitting its signals through the frequency of $915 \mathrm{M}$, it is utilizing its bandwidth. During this, as we already defined the primary users as the highest priority user, none of the secondary user allows to uses that frequency and also they are not even allow to generate the request to the primary users.

Table.1. shows the configurations of the waveform

\begin{tabular}{|l|l|}
\hline \multicolumn{2}{|l|}{ CONFIGURATIONS } \\
\hline $\begin{array}{l}\text { CARRIER } \\
\text { FREQUENCY }\end{array}$ & $915 \mathrm{MHz}$ \\
\hline IQ RATE & $400 \mathrm{k}$ \\
\hline GAIN & 0 \\
\hline
\end{tabular}

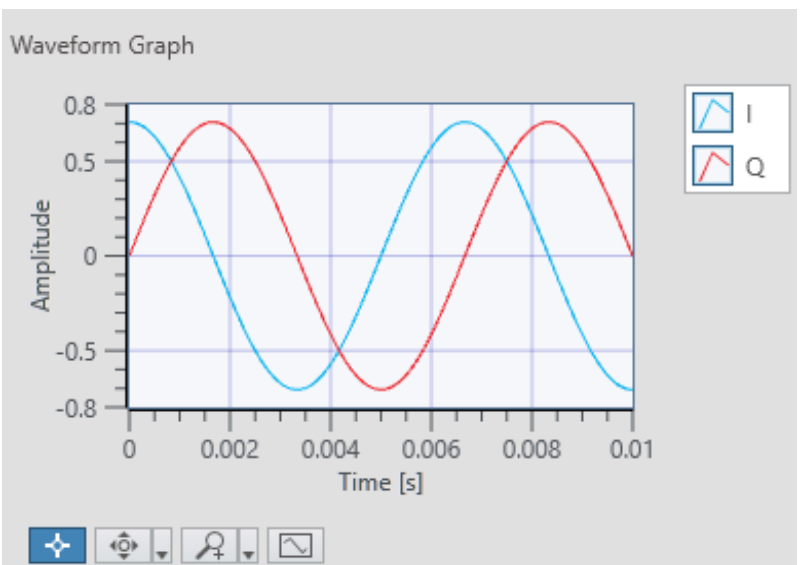

Fig.6. shows the waveform transmitted by the primary user. 


\section{b) Secondary user monitors the signals}

In fig.7, during the primary user transmission, the secondary user is continuously monitoring the peak generates by the primary during the signal transmission. We designed the system in such a way that when the secondary receives the value 0 , i.e. there is no peak generated by primary user. So, to utilize that bandwidth, the secondary user generates the request to the primary.

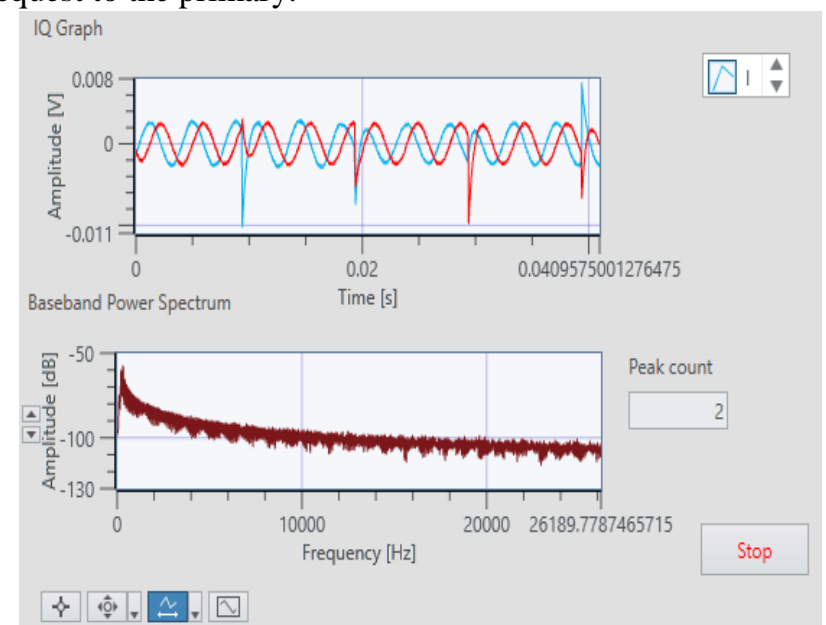

Fig.7. shows the signal detected by the secondary user.

\section{c) Secondary user transmits the signal}

Fig.8. shows, we designed our system in such a way that the request generated by secondary is of specific format like:

U1: 915Hz; here, theU indicates the starting of the address which helps the system to understand the address of the secondary user starts after ' $U$ ' and ends before'; '. So, after generating of this message request, the message bits are arranged it is transmitted to the primary for the further process.

Table.2. shows the configuration of the signal transmitted by the secondary user.

\begin{tabular}{|c|c|}
\hline \multicolumn{2}{|c|}{ CONFIGURATIONS } \\
\hline $\begin{array}{c}\text { TxCARRIER } \\
\text { FREQUENCY }\end{array}$ & $915 \mathrm{MHz}$ \\
\hline Tx IQ RATE & $1 \mathrm{M}$ \\
\hline Tx GAIN & 0 \\
\hline
\end{tabular}

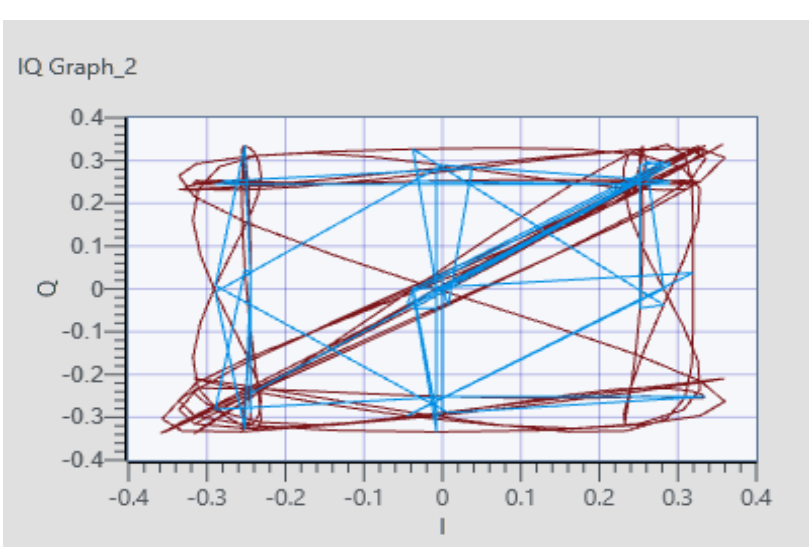

Fig.8. shows the signal of the message transmitted by the secondary user.

\section{d) Primary user receives the message}

In fig.9, at the receiving end of the primary user after receiving the request, the system designed by us is in such a way that firstly, it check that the received message string is empty or not. If it's in not just an empty string, the system checks the first index of that string and compare it with the ' $U$ ', if the string starts with the ' $U$ ', then only it's considered as an address otherwise it is just a random string for the system. After finding the ' $U$ ', then the system starts scanning the string until its gets the first ';', and between the ' $U$ ' and the ';', its considered it as an address of the secondary user, and then after getting the first ';', the system starts searching for the next ';'. After getting the last ';', the system excludes that ';' and gets the frequency which is requested by the secondary user. Now, if the primary want to allow the secondary user to use that bandwidth, then if starts arranging all the received requests in a stack to track them, and then allow the desired time to all the desired secondary users with the free time available for the particular spectrum. In our research, we designed the system in such a way, that it allotted two hours to multiple secondary users and then after the ending time of each user, we allotted five minutes difference before the start time of the next user. During the time allotment for the whole particular day, the system is designed that it didn't allow any further request after 22:00. And is still the primary received any request after 22:00, then that secondary request denied.

Table.3. shows the configurations of the received message

\begin{tabular}{|l|l|}
\hline \multicolumn{2}{|c|}{ CONFIGURATIONS } \\
\hline $\begin{array}{l}\text { Rx CARRIER } \\
\text { FREQUENCY }\end{array}$ & $915 \mathrm{MHz}$ \\
\hline Rx IQ RATE & $400 \mathrm{k}$ \\
\hline Rx GAIN & 28 \\
\hline $\begin{array}{l}\text { MODULATION } \\
\text { SCHEME }\end{array}$ & BPSK \\
\hline MESSAGE BITS & 128 \\
\hline
\end{tabular}

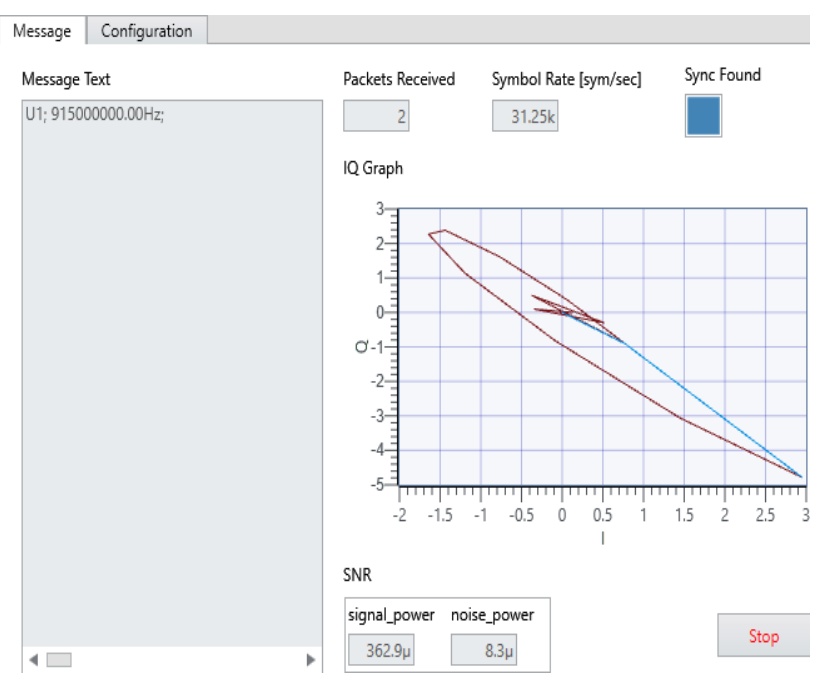

Fig.9. shows the message received by the primary user.

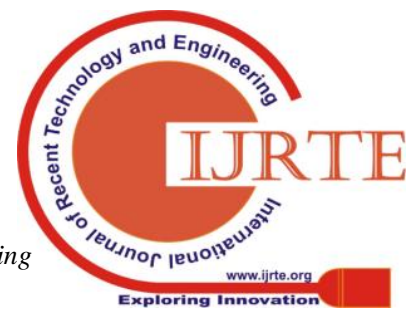




\section{CONCLUSION AND FUTURE SCOPE}

Current research uses the primary user with the single frequency, which is to be sensed and considered as an available frequency for secondary user. All the transmission and reception are done for $915 \mathrm{MHz}$ frequency only. The results are satisfactory for the testbed design for the small scale network. In future research the work will extended for the band of multiple frequencies, and to design the primary premises in such a way that the primary user should be able to allot the frequency on the demand of secondary user in that multiple frequency band. The primary user should also have to manage the secondary users request along with the time duration and cost for that allotted time interval.

\section{REFERENCES}

1. FCC, ET Docket No 03-322 Notice of Proposed Rule Making and Order, Dec 2003.

2. I. F. Akyildizet al., "NeXt Generation/ Dynamic Spectrum Access/Cognitive Radio Wireless Networks: A Survey," Comp. Networks J., vol. 50, Sept. 2006, pp. 2127-59.

3. S. Haykin, "Cognitive Radio: Brain Empowered Wireless Communications," IEEE JSAC, vol. 23, no. 2, Feb. 2005, pp. 201-20.

4. F. K. Jondral, "Software-Defined Radio - Basic and Evolution to Cognitive Radio," EURASIP J. WirelessCommun. and Networking, 2005.

5. I. F. Akyildiz, W.-Y. Lee, and K. Chowdhury, "CRAHNs: Cognitive Radio Ad Hoc Networks," Ad Hoc Net. J., vol. 7, no. 5, July 2009.

6. O. Ileri, D. Samardzija, and N. B. Mandayam, "Demand Responsive Pricing and Competitive Spectrum Allocation via Spectrum Server," Proc. IEEE DySPAN 2005, Nov. 2005, pp. 194-202.

7. M. Oner and F. Jondral, "On the Extraction of the Channel Allocation Information in Spectrum Pooling Systems," IEEEJSAC, vol. 25, no. 3, Apr. 2007, pp. 558-65.

8. S. M. Mishra, A. Sahai, and R. W. Brodersen, "Cooperative Sensing among Cognitive Radios," Proc. IEEE ICC2006, vol. 4, June 2006, pp. 1658-63.

9. B. Wild and K. Ramchandran, "Detecting Primary Receivers for Cognitive Radio Applications," Proc. IEEEDySPAN 2005, Nov. 2005, pp. 124-30.

10. S. Krishnamurthy et al., "Control Channel Based MACLayer Configuration, Routing and Situation Awareness for Cognitive Radio Networks," Proc. IEEE MILCOM2005, Oct. 2005, pp. 455-60. 